\title{
Distúrbios osteomusculares relacionados ao trabalho: uma caracterização da sintomatologia e dos exames diagnósticos
}

\author{
Work-related musculoskeletal disorders: a characterization of symptomatology and diagnostic \\ exams
}

Trastornos osteomusculares en el trabajo: una caracterización de los exámenes de sintomatología y diagnóstico

Recebido: 11/01/2021 | Revisado: 12/01/2021 | Aceito: 15/01/2021 | Publicado: 18/01/2021

Iraneide Nascimento dos Santos
ORCID: https://orcid.org/0000-0001-8449-7840
Instituto Federal de Educação, Ciência e Tecnologia de Pernambuco, Brasil
E-mail: iraneidenascimento@ipojuca.ifpe.edu.br
Islane Cristina Martins
ORCID: https://orcid.org/0000-0003-2351-2730
Universidade Federal de Pernambuco, Brasil
E-mail: relacionamento@ @assenomestrado.com
Amilton da Cruz Santos
ORCID: https://orcid.org/0000-0003-3856-4197
Universidade Federal da Paraíba, Brasil
E-mail: adagatom@ yahoo.com.br
Adriana Sarmento de Oliveira
ORCID: https://orcid.org/0000-0002-7680-0958
Universidade Anhembi Morumbi, Brasil
E-mail: adriana.sarmento@ alumni.usp.br
Maria do Socorro Brasileiro-Santos
ORCID: https://orcid.org/0000-0001-7175-4078
Universidade Federal da Paraíba, Brasil
E-mail: sbrasileiro@yahoo.com.br

\section{Resumo}

Objetivo: caracterizar as queixas clínicas e os achados de imagem nos distúrbios osteomusculares relacionados ao trabalho (DORT). Método: trata-se de uma pesquisa descritiva, exploratória e transversal, realizada em um Centro de Referência à Saúde do Trabalhador, com uma amostra composta de 60 sujeitos. Foi realizada uma entrevista individual, que utilizou um questionário e um roteiro para análise dos exames. Resultados: a população foi em sua maioria masculina $(66,7 \%)$, a profissão mais acometida foi o operador de máquina $(18,3 \%)$, as queixas mais relatadas foram dor $(100 \%)$ e falta de força $(93,3 \%)$. Os locais mais citados foram o ombro direito $(26,5 \%)$ e a coluna lombar $(37,5 \%)$. Os diagnósticos mais prevalentes foram as tendinopatias nos ombros $(39,1 \%)$ e os transtornos nos discos vertebrais $(28,1 \%)$. Os exames mais utilizados para auxiliar no diagnóstico foram a ultrassonografia $(38 \%)$ e a ressonância $(36,5 \%)$. As alterações mais encontradas nos tendões, músculos, sinóvias e bursas - inflamações $(10,3 \%)$ e calcificações $(11,2 \%)$ - e nos discos vertebrais - protusões discais $(13,1 \%)$ e presença de osteófitos $(10,3 \%)$. Considerou-se um nível de significância $\mathrm{p}<0,05$ e intervalo de confiança (IC) de $95 \%$ para os cálculos das frequências. Conclusão: tendo em vista a vulnerabilidade dos trabalhadores aos distúrbios, é importante valorizar as queixas clínicas do paciente e as informações contidas nos exames de imagem, com a finalidade de se ter o diagnóstico clínico precoce e de auxiliar os profissionais de saúde a viabilizarem a terapêutica mais adequada.

Palavras-chave: Doenças musculoesqueléticas; Sinais e sintomas; Dor musculoesquelética; Fatores de risco; Diagnóstico por imagem.

\footnotetext{
Abstract

Objective: this study aims to characterize the clinical complaints and imaging findings in musculoskeletal disorders related to work (RSI). Method: this is a descriptive, exploratory, and cross-sectional research, conducted from October 2008 to September 2009 in a Reference Center for Occupational Health, with a sample of 60 subjects. We performed a separate interview, using a questionnaire and a roadmap for analysis of tests. Results: the population was mostly male $(66.7 \%)$, the profession most affected was the machine operator $(18.3 \%)$, the most frequently reported complaints were pain (100\%) and lack of strength (93.3\%). The most cited were the shoulder D (26.5\%) and lumbar spine (37.5\%). The most used tests for the diagnosis were ultrasonography (38\%) and magnetic resonance $(36.5 \%)$. The most found changes in tendons, muscles, synovias and bursas - inflammations (10.3\%) and calcifications (11.2\%)
} 
- and in vertebral discs - disc protusons (13.1\%) and presence of osteophytes (10.3\%). A significance level p<0.05 and confidence interval (CI) of $95 \%$ were considered for the calculations of frequencies. Conclusion: in view of the vulnerability of workers to disorders, it is important to value the patient's clinical complaints and the information contained in imaging exams, with the purpose of having an early clinical diagnosis and assisting health professionals to enable the most appropriate therapy.

Keywords: Musculoskeletal diseases; Signs and symptoms; Musculoskeletal pain; Risk factors; Diagnostic imaging.

\section{Resumen}

Objetivo: caracterizar las quejas clínicas y los hallazgos de imágenes en trastornos musculoesqueléticos relacionados con el trabajo (TSD). Método: se trata de una investigación descriptiva, exploratoria y transversal llevada a cabo en un Centro de Referencia para la Salud de los Trabajadores, con una muestra compuesta por 60 asignaturas. Se realizó una entrevista individual, que utilizó un cuestionario y un guión para analizar los exámenes. Resultados: la población era mayoritariamente masculina $(66,7 \%)$, la profesión más afectada fue el operador de la máquina $(18,3 \%)$, las quejas más notificadas fueron dolor $(100 \%)$ y falta de fuerza $(93,3 \%)$. Los sitios más citados fueron el hombro derecho $(26,5 \%)$ y la columna lumbar (37,5\%). Los diagnósticos más frecuentes fueron tendinopatías en los hombros $(39,1 \%)$ y trastornos del disco vertebral $(28,1 \%)$. Las pruebas más utilizadas para ayudar en el diagnóstico fueron la ecografía $(38 \%)$ y resonancia $(36,5 \%)$. Los cambios más comúnmente encontrados en tendones, músculos, sinovias y bursas inflamaciones $(10.3 \%)$ y calcificaciones $(11,2 \%)$ - y en discos vertebrales - protusones de disco $(13,1 \%)$ y la presencia de osteofitos $(10,3 \%)$. Para los cálculos de frecuencias se consideró un nivel de significancia $\mathrm{p}<0,05 \mathrm{y}$ un intervalo de confianza (CI) del 95\%. Conclusión: en vista de la vulnerabilidad de los trabajadores a los trastornos, es importante valorar las quejas clínicas del paciente y la información contenida en los exámenes por imágenes, con el propósito de tener un diagnóstico clínico temprano y ayudar a los profesionales de la salud a permitir la terapia más adecuada.

Palabras clave: Enfermedades musculoesqueléticas; Signos y síntomas; Dolor musculoesquelético; Factores de riesgo; Diagnóstico por imagen.

\section{Introdução}

As lesões agudas relacionadas ao trabalho e os distúrbios osteomusculares relacionados ao trabalho são um dos problemas mais comuns entre os trabalhadores mundialmente (Organização Internacional do Trabalho, 2018; Skovlund et al., 2020), e continuam sendo uma importante causa de incapacidades laborais e dias de trabalho perdidos (Organização Internacional do Trabalho, 2018).

Nesse sentido, os distúrbios osteomusculares relacionados ao trabalho - DORT são danos decorrentes da utilização excessiva do sistema osteomuscular, e da falta de tempo para recuperação das estruturas envolvidas (Ministério da Saúde, 2012, 2019; Souza et al., 2020).

Ainda, os DORT são caracterizados pela ocorrência de vários sintomas nas estruturas osteomioarticulares, predominantemente nos membros superiores, tais como: dor, parestesia, sensação de peso e fadiga, que surgem de forma insidiosa, concomitantes ou não, podem interagir entre si e devem ser sempre avaliados integralmente (Ministério da Saúde, 2012, 2019).

Além disso, esses sintomas são resultantes de vários fatores que podem predispor os trabalhadores às lesões musculoesqueléticas, incluindo políticas e práticas organizacionais, fatores predisponentes presentes no ambiente de trabalho, das características do trabalhador e do grupo ocupacional a que pertence (Orange et al., 2016).

Assim, os fatores mencionados, os fatores biomecânicos e os psicossociais são os principais agentes motivadores (Orange et al., 2016; Alireza et al., 2020). Logo, são considerados os grandes preditores do surgimento de quadros álgicos nos DORT a movimentação manual, o trabalho repetitivo e o estático, as vibrações e as condições psicológicas e as sociais precárias entre trabalhadores expostos (Ministério da Saúde, 2019).

Desse modo, em virtude do caráter multifatorial e de frequentemente não haver sinais objetivos que caracterizem esses distúrbios, o diagnóstico é de difícil identificação, e muitas vezes o paciente pode não apresentar nenhum sinal físico inicialmente, embora suas queixas sejam persistentes e condizentes com a sintomatologia (Pereira, 2015). 
Logo, é importante que o médico realize uma anamnese que deve incluir a história clínica detalhada, o exame físico, o diagnóstico diferencial e os exames de imagem mais indicados para as queixas relatadas pelo paciente (Amorim, 2006; Ministério da Saúde, 2012).

Nessa perspectiva, vários exames têm sido utilizados para fazer o diagnóstico de DORT, a saber: radiografia, ultrassonografia, tomografia computadorizada, eletroneuromiografia e ressonância magnética (Pereira, 2015). Dentre estes, a ultrassonografia é usada com frequência, embora a ressonância magnética tenha uma maior sensibilidade e especificidade para a maioria desses distúrbios, contudo, ainda é menos utilizada em decorrência do alto custo (Pereira, 2015).

E, mesmo que a ultrassonografia seja a mais utilizada, no Brasil, ela é pouco solicitada para diagnóstico de lesões relacionadas ao trabalho em exames ocupacionais, tais como: admissional, mudança de função, retorno ao trabalho, periódico, e demissional, o que auxiliaria na orientação do melhor posto de trabalho para inserir o laborioso, como também, dificulta o acompanhamento do histórico laboral do trabalhador quanto às exposições às condições adversas ao longo da vida (Pereira, 2015).

Salienta-se ainda que, as Lesões por Esforços Repetitivos (LER) e os Distúrbios Osteomusculares Relacionados ao Trabalho (DORT) são as doenças que mais afetam os trabalhadores brasileiros. Além disso, 134.670 casos de LER/DORT foram notificados pelo SINAN entre 2007 e 2018, neste período, o total de registros cresceu 506\%, passando de 3.433 casos, em 2007, para 17.392 em 2018 (Ministério da Saúde, 2019). Dessa forma, tanto o volume quanto o aumento nos casos destacam a gravidade do problema, que afeta a saúde dos trabalhadores (Organização Internacional do Trabalho, 2018). Ainda, os estudos que abordam o assunto com enfoque nas queixas clínicas e nos achados de exames de imagem são insipientes, e, por isso, há a necessidade de se pesquisar acerca do assunto com o fim de auxiliar os profissionais de saúde que lidam diretamente com o problema, de reduzir o impacto social e os prejuízos que os distúrbios acarretam aos trabalhadores, às empresas (Lopes et al., 2017), ao sistema de saúde pública e à previdência social (Freitas et al., 2015).

Diante do exposto, o objetivo do presente estudo foi caracterizar as queixas clínicas e os achados de imagem nos distúrbios osteomusculares relacionados ao trabalho de trabalhadores atendidos em um Centro de Referência em Saúde do Trabalhador.

\section{Metodologia}

Trata-se de um estudo descritivo, exploratório, de corte transversal, pois descreve um fenômeno delimitando-se o momento no qual a população foi examinada (Zangirolami-Raimundo, Echeimberg, \& Leone, 2018), e com abordagem quantitativa, visto que quantificou os dados objetivos através da coleta de informações de questionários que foram analisados com o emprego de técnicas estatísticas (Pereira et al., 2018).

A pesquisa foi desenvolvida no Centro de Referência em Saúde do Trabalhador (CEREST) localizado no Município do Cabo de Santo Agostinho - Pernambuco. Atualmente ele é um centro regional, com função de prover o suporte técnico, auxiliar nos processos de capacitação e educação permanente em Saúde do Trabalhador para participantes do controle social, profissionais e técnicos da rede do SUS, todavia, no período da pesquisa, atendia a todos os trabalhadores da cidade do Cabo e cidades circunvizinhas, como Amaragi, Barra de Guabiraba, Barreiros, Chã Grande, Cortez, Escada, Gameleira, Ipojuca, Joaquim Nabuco, Primavera, Ribeirão, Rio Formoso, São José da Coroa Grande, Sirinhaém e Tamandaré, que poderiam chegar por demanda espontânea ou encaminhados de sindicatos, de unidades de saúde da prefeitura, de advogados, entre outros.

A população deste estudo teve uma amostra contendo 60 participantes, de ambos os sexos, com diagnóstico clínico dos DORT, e por conveniência, justificada pela homogeneidade da população de trabalhadores com distúrbios atendidos por 
demanda espontânea no CEREST Cabo de Santo Agostinho, com relação às características socioculturais, socioeconômicas, ocupacionais e clínicas.

Foram excluídos os indivíduos com história de acidente de trabalho típico, ou seja, acidentes que aconteceram durante as atividades habituais do trabalho; com alterações osteomusculares devido a atividades fora do ambiente de trabalho; com história de doenças articulares, musculares, vasculares e reumáticas; em uso de antibióticos recentemente; com malformações congênitas; ou com outras doenças que não sejam os DORT.

Este estudo atendeu aos aspectos éticos das pesquisas que envolvem seres humanos do Conselho Nacional de Saúde e foi aprovada ao Comitê de Ética em Pesquisa com Humanos da Universidade Federal de Pernambuco, sob o $n^{\circ}$ 0240.0.172.000-08. Vale ressaltar que, antes da realização da entrevista, foi pedida a autorização dos voluntários através do termo de consentimento livre e esclarecido.

O instrumento utilizado nessa pesquisa continha 30 questões objetivas e foi adaptado do roteiro de investigação de LER/DORT do Ministério da Saúde (2001). O questionário apresentou três partes, a primeira foi constituída de variáveis relativas aos fatores individuais (idade, sexo, peso, altura); na segunda parte foram abordados os riscos ocupacionais; e na terceira, as alterações osteomusculares. Além do questionário, utilizou-se um formulário, construído pelos autores, para registrar as informações encontradas nos exames de imagem.

$\mathrm{Na}$ análise descritiva as variáveis foram distribuídas em frequências absolutas (n) e relativas (\%), gráficos e tabelas. Foram realizados os cálculos das frequências através de medidas como a média, a moda, o máximo, o mínimo e o desvio padrão, onde considerou-se um nível de significância p < 0,05 e intervalo de confiança (IC) de 95\%.

Não foram realizados testes específicos baseados em associações em decorrência do tipo de estudo e variáveis utilizados. O software utilizado foi o Excel 2000 e o programa BioEstat 5.

\section{Resultados}

Foram entrevistados 60 voluntários, com idade mínima e máxima, de 23 e 59 anos, respectivamente, que se distribuíram na seguinte ordem crescente: 31 e 40 anos (38,3\%); 41 e 50 anos (26,7\%); 23 e 30 anos (23,3\%) e 51 e 59 anos (11,7\%), com média de idade de 38,3 9,2 anos. Em relação ao gênero, 66,7\% da amostra é do sexo masculino.

No tocante às funções laborais consideradas principais pela maioria da amostra, 18,3\% eram operadores de máquina, $15 \%$ ajudantes de produção e $10 \%$ auxiliares de serviços gerais, nas quais, as duas primeiras profisssões com maior prevalência são essencialmente do setor industrial.

Já, as demais profissões, tais como: auxiliar administrativo, auxiliar de carga e descarga, auxiliar de cozinha, auxiliar de expedição, auxiliar de impressão, camareira, copeira, costureira, embaladora, empilhador, manipulador, mecânico, monitor de qualidade, motorista, operador de caixa, pedreiro, pintor, secretária, soldador, tecelã, técnica de enfermagem, técnico gráfico, torneiro mecânico, vendedora, vigilante, tiveram um percentual menor que $10 \%$ cada. 
Figura 1. Distribuição percentual das queixas osteomiarticulares (painel A) e locais do corpo que as incidiram nos trabalhadores com DORT (painel B). D: direito; E: esquerdo.
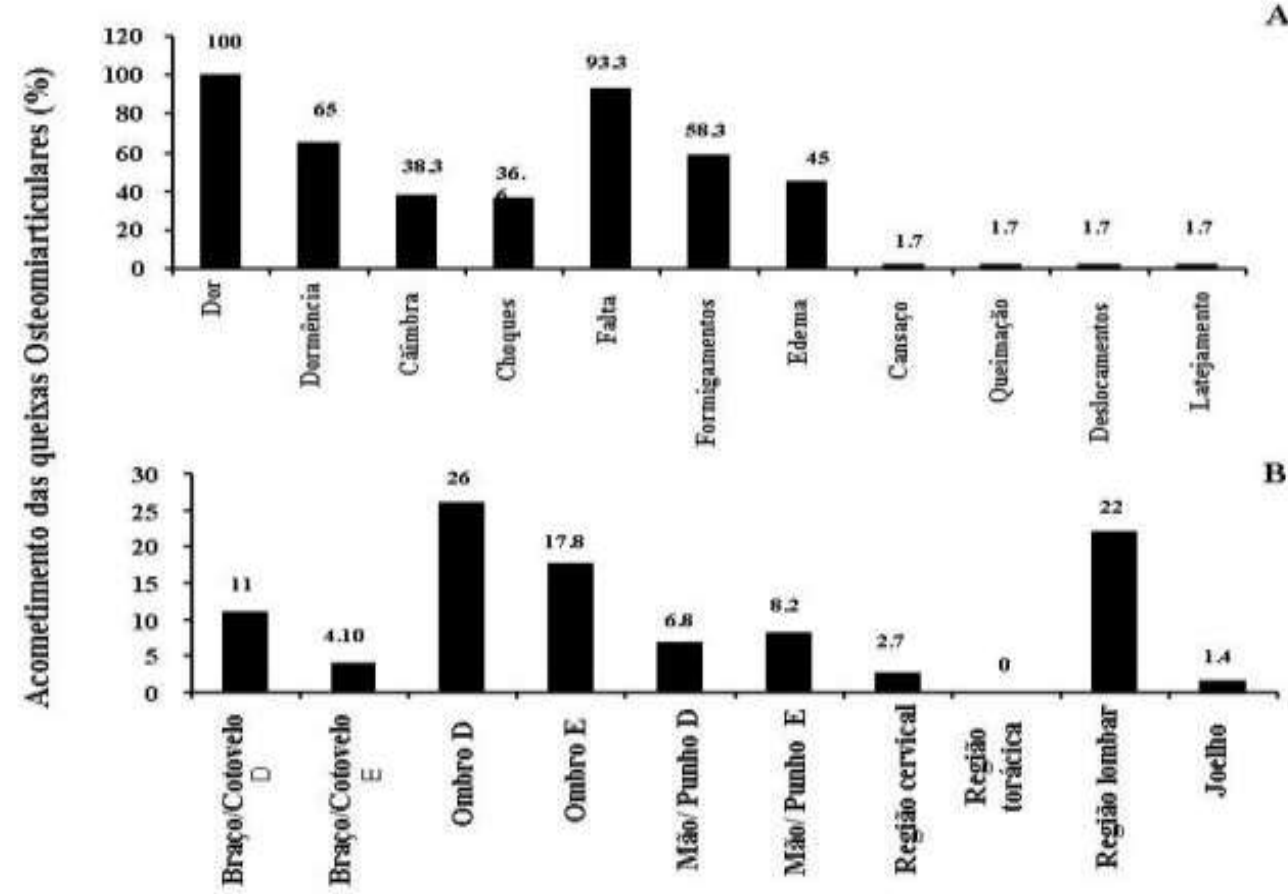

Fonte: Autores.

No entanto, independente da função, $100 \%$ dos entrevistados relataram sintomatologia dolorosa, 93,3\% falta de força, $65 \%$ dormência, $58,3 \%$ formigamentos, $45 \%$ edema, 38,3\% câimbra, 36,6\% choques, e cansaço, queimação, deslocamentos e latejamento, menos de 10\% cada um (Figura 1; painel A). Além disso, as queixas descritas estavam presentes há menos de cinco anos para 88,3\% dos entrevistados, entre cinco e dez anos para 8,3\% e há mais de dez anos para 3,3\%.

No que tange às regiões do corpo, apresentaram as maiores incidências dessas queixas osteomiarticulares o ombro direito $(17,8 \%)$ e a região lombar $(22 \%)$ (Figura 1; painel B). Contudo, nenhum trabalhador referiu queixas na região torácica da coluna vertebral.

A respeito dos diagnósticos de DORT, o mais prevalente na amostra foi de alterações na região do ombro, como mostra o painel B da Figura 1, entretanto o seu total não corresponde ao tamanho da amostra estudada, uma vez que alguns tiveram mais de uma hipótese diagnóstica. 
Tabela 1. Distribuição dos diagnósticos de DORT.

\begin{tabular}{c|cc}
\hline DIAGNÓSTICOS & \multicolumn{2}{|c}{ FREQUÊNCIAS } \\
& Absoluta & Relativa (\%) \\
\hline Bursite no ombro & 4 & 6,2 \\
Tendinopatia subescapular, supra-espinhal, infraescapular, calcária, síndrome & 25 & 39,1 \\
do manguito rotador e osteoartrose acromioclavicular & 2 & 3,1 \\
Tendinopatia De Quervain & 2 & 7,8 \\
Epicondilite lateral e/ou medial & 5 & 7,8 \\
Síndrome do túnel do carpo & 5 & 1,6 \\
Cisto sinovial & 1 & 4,7 \\
Sinovite e tenossinovite no punho & 3 & 28,1 \\
Transtornos nos discos vertebrais & 18 & 1,6 \\
Convicalgias, lombalgias, ciatalgias, osteoartrose) & & 100 \\
\hline
\end{tabular}

Fonte: Autores.

Nesse estudo, 39,1\% da amostra teve hipótese diagnóstica de tendinopatia subescapular, supra-espinhal, infraescapular, calcária e/ou osteoartrose acromioclavicular, 28,1\% apresentaram transtornos nos discos vertebrais como as degenerações, os deslocamentos com e sem compressão nervosa, e a osteoartrose (Tabela 1). Nessa tabela o total é diferente do tamanho da amostra estudada porque houve mais de uma hipótese diagnóstica.

Tabela 2. Associação entre as queixas clínicas e o gênero em trabalhadores com DORT.

\begin{tabular}{|c|c|c|}
\hline \multirow{2}{*}{ QUEIXAS CLÍNICAS } & \multicolumn{2}{|c|}{ GÊNERO (\%) } \\
\hline & Masculino & Feminino \\
\hline Dor & 23,7 & 20,4 \\
\hline Dormência & 15,9 & 12,2 \\
\hline Câimbras & 8,3 & 19,1 \\
\hline Falta de força & 20,7 & 19,3 \\
\hline Edema & 6,5 & 16,3 \\
\hline Formigamentos & 13,6 & 14,2 \\
\hline Choques & 8,8 & 8,1 \\
\hline Queimação & 0,6 & 1,0 \\
\hline Latejamento & 0,6 & - \\
\hline Cansaço & 0,6 & - \\
\hline Deslocamentos & 0,6 & - \\
\hline Total & 100 & 100 \\
\hline
\end{tabular}


$\mathrm{Na}$ estatística descritiva primeiramente foi feita a associação entre o gênero e as queixas clínicas como pode ser visualizada abaixo, na Tabela 2. E, verificou-se que em relação às queixas apresentadas, a diferença não foi significante, uma vez que os valores se aproximaram nas queixas de dor, dormência, falta de força, choques e formigamentos.

Ainda em relação às queixas clínicas, houve uma diferença significativa para câimbra e presença de edema, mais prevalentes no sexo feminino. Como também, as queixas de cansaço, latejamento e deslocamento foram apresentadas apenas pelo sexo masculino.

Tabela 3. Distribuição sobre os exames de imagem, regiões corporais, presença de alterações patológicas e período de realização desses exames nos trabalhadores com DORT.

\begin{tabular}{|c|c|c|}
\hline EXAMES REALIZADOS & n & $\%$ \\
\hline Raio-X & 6 & 9,5 \\
\hline Ultrassonografia & 24 & 38 \\
\hline Tomografia & 2 & 3,2 \\
\hline Ressonância & 23 & 36,5 \\
\hline Eletroneuromiografia & 4 & 6,4 \\
\hline Total & 60 & 100 \\
\hline Locais do corpo descritos nos achados de imagem & $\mathbf{N}$ & $\%$ \\
\hline Punho/mão & 10 & 17 \\
\hline Cotovelo & 4 & 6,8 \\
\hline Ombro & 25 & 42,4 \\
\hline Região cervical & 2 & 3,4 \\
\hline Região lombar & 17 & 28,8 \\
\hline Joelho & 1 & 1,7 \\
\hline Total & 60 & 100 \\
\hline Realizaram Exames de Imagem & $\mathbf{N}$ & $\%$ \\
\hline Sim & 56 & 93,3 \\
\hline Não & 4 & 6,6 \\
\hline Total & 60 & 100 \\
\hline Presença de alterações nos exames de imagem & $\mathbf{N}$ & $\%$ \\
\hline Sim & 50 & 89,3 \\
\hline Não & 6 & 10,7 \\
\hline Total & 56 & 100 \\
\hline Data da realização dos exames em meses & $\mathbf{N}$ & $\%$ \\
\hline$<$ três & 23 & 41 \\
\hline três - seis & 9 & 16,1 \\
\hline seis - nove & 7 & 12,5 \\
\hline nove - doze & 5 & 8,9 \\
\hline$>$ doze & 12 & 21,4 \\
\hline Não fizeram exame & 4 & 6,7 \\
\hline Total & 60 & 100 \\
\hline
\end{tabular}

Fonte: Autores. 
$\mathrm{Na}$ análise da Tabela 3 verificou-se que os exames imaginológicos de escolha foram a ultrassonografia e a ressonância magnética, com $38 \%$ e 36,5\% respectivamente, os demais exames como os raios-X, a tomografia computadorizada e eletroneuromiografia tiveram menos de $10 \%$ cada. Além disso, as regiões do corpo que tiveram as maiores prevalências de realização dos exames foram o ombro (42,4\%), a coluna lombar (28,8\%) e a mão/punho (17\%).

Além disso, 93,3\% dos 60 trabalhadores que tiveram o exame de imagem solicitado, as alterações patológicas foram encontradas em $89 \%$ destes. E, quanto à data da realização dos exames, $41 \%$ realizaram a menos de três meses, $21,4 \%$ acima de doze meses, $16,1 \%$ entre três e seis meses, $12,5 \%$ entre seis e nove meses e $8,9 \%$ entre nove e dez meses (Tabela 3).

Tabela 4. Distribuição das alterações patológicas encontradas nas estruturas corporais segundo os exames de imagem dos trabalhadores com DORT.

\begin{tabular}{|c|c|c|c|}
\hline \multirow{2}{*}{$\begin{array}{c}\text { ESTRUTURAS } \\
\text { CORPORAIS }\end{array}$} & \multirow[t]{2}{*}{ ALTERAÇÕES PATOLÓGICAS } & \multicolumn{2}{|c|}{ FREQUÊNCIAS } \\
\hline & & Absoluta & Relativa (\%) \\
\hline Tendões Músculos & Inflamatórias & 11 & 10,3 \\
\hline \multirow[t]{12}{*}{ Sinóvias e Bursas } & Calcificações & 12 & 11,2 \\
\hline & Fibrose & 6 & 5,6 \\
\hline & Presença de Líquido & 6 & 5,6 \\
\hline & Espessamentos & 9 & 8,4 \\
\hline & Heterogeneidade Estrutural & 1 & 0,9 \\
\hline & Hipoecogenicidade & 3 & 2,8 \\
\hline & Estrutura com Calibre Aumentado & 2 & 1,8 \\
\hline & Estrutura com Calibre Diminuído & 1 & 0,9 \\
\hline & Fissuras & 1 & 0,9 \\
\hline & Compressões Miotendíneas & 2 & 1,8 \\
\hline & Rupturas Parciais & 1 & 0,9 \\
\hline & Cistos & 1 & 0,9 \\
\hline \multirow[t]{7}{*}{ Discos Vertebrais } & Desidratação dos Discos Vertebrais & 5 & 4,7 \\
\hline & Abaulamentos Discais & 1 & 0,9 \\
\hline & Protusões Discais & 14 & 13,1 \\
\hline & Osteófitos & 11 & 10,3 \\
\hline & Discopatia Degenerativa & 2 & 1,8 \\
\hline & Redução dos Espaços Discais & 3 & 2,8 \\
\hline & Presença de Hérnia Intra-Esponjosa & 1 & 0,9 \\
\hline \multirow[t]{2}{*}{ Articulações } & Hipoplasia de Facetas Articulares & 1 & 0,9 \\
\hline & Distensão da Cápsula Articular & 1 & 0,9 \\
\hline \multirow[t]{2}{*}{ Ossos } & Condromalácia & 1 & 0,9 \\
\hline & Irregularidade na Superfície Óssea & 1 & 0,9 \\
\hline \multirow[t]{2}{*}{ Nervos } & Redução de Condução Sensitiva & 3 & 2,8 \\
\hline & Compressão de Raiz Nervosa & 7 & 6,5 \\
\hline
\end{tabular}


Acerca das alterações patológicas encontradas nas estruturas corporais segundo os exames de imagem dos trabalhadores, o maior número delas foi identificado nos tendões, músculos, sinóvias e bursas - inflamações (10,3\%) e calcificações $(11,2 \%)$ - e nos discos vertebrais - protusões discais $(13,1 \%)$ e presença de osteófitos $(10,3 \%)$ (Tabela 4).

Ainda em relação aos resultados de imagem, nos exames dos operadores de máquina, que é a função com maior prevalência na amostra, as estruturas mais identificadas foram: tendão $(58,3 \%)$ e discos vertebrais $(25 \%)$ com predomínio de calcificações, fibroses, espessamentos e protusões discais.

E, a ultrassonografia e a ressonância magnética foram os exames mais solicitados para as estruturas do tendão e dos discos vertebrais, respectivamente.

\section{Discussão}

O presente estudo que buscou caracterizar as queixas clínicas e os achados de imagem nas doenças osteomusculares relacionadas ao trabalho, identificou uma amostra jovem, masculina, essencialmente do setor industrial, com 100\% de prevalência de dor há menos de cinco anos, com queixas localizadas em ombro direito e região lombar, nas quais as hipóteses diagnósticas mais encontradas foram as tendinopatias e os transtornos nos discos vertebrais, sendo os exames mais utilizados para auxiliar no diagnóstico, a ultrassonografia e a ressonância.

Nesse sentido, no tocante à faixa etária os voluntários apresentaram em sua maioria idade entre 31 e 40 anos (38,3\%), assim como em outros estudos acerca dos DORT (Silva Júnior, Buzzoni \& Morrone, 2016; Silva et al., 2019). Isso demonstra que os distúrbios osteomioarticulares surgem quando os trabalhadores estão no ápice da vida produtiva, logo, esses indivíduos economicamente ativos estão sendo vítimas de doenças e acidentes do trabalho, levando a perdas sociais e econômicas ao país (Trindade et al., 2014).

Como também, os dados apontam que a segunda faixa etária com maior percentual foi entre 41 e 50 anos (26,7\%), pois esses distúrbios se apresentam e ou se agravam com o processo de envelhecimento, promovendo a degradação progressiva da função mioarticular e das medidas antropométricas, que altera a força e a flexibilidade musculares (Trindade et al., 2014), o que destaca a importância que deve ser dada a prevalência dos DORT nesse intervalo de idades.

Além disso, em indivíduos com idade superior a 35 anos podem surgir principalmente as lesões degenerativas nos tendões, pois a reposição do tecido tendíneo já não é tão eficaz, tanto no que diz respeito à velocidade quanto à qualidade (Lida \& Guimarães, 2016). Adicionalmente, as alterações no arcabouço físico das estruturas e na microvascularização ocorridas em virtude do processo de envelhecimento, podem receber influências hormonais, bioquímicas, imunológicas e mecânicas (Lida \& Guimarães, 2016).

Também, acredita-se que esse processo se inicie por volta dos trinta a quarenta anos, acelerando-se a partir dos cinquenta anos (Trindade et al., 2014; Carvalho, Brasileiro \& Lima, 2019), portanto, a morbidade musculoesquelética é mais esperada em idades mais avançadas, a menos que outros fatores de risco desencadeiem esse problema mais precocemente (Carvalho, Brasileiro \& Lima, 2019).

Conforme foi mencionado, os fatores biomecânicos como as posturas e os movimentos inadequados, assim como, a carga estática e dinâmica, além da ausência de pausas entre as atividades, predispõem os indivíduos às lesões musculoesqueléticas (Lida \& Guimarães, 2016; Souza et al., 2020).

E, mesmo que esses indivíduos sejam significativamente diferentes em suas funções fisiológicas, capacidade cardiovascular, forças musculares e dimensões antropométricas, eles estão igualmente susceptíveis às consequências danosas que as condições inseguras e precarizadas do trabalho impõem aos homens e mulheres (Rosa \& Quirino, 2018).

Nessa vertente, observou-se nesse estudo um maior acometimento das patologias no sexo masculino (66,7\%). Isto deve-se ao fato de o perfil profissional da amostra ser industrial, com maior número de operadores de máquina (18,3\%) e 
ajudantes de produção (15\%), como também, nesse ramo produtivo, normalmente se empregam homens (Rosa \& Quirino, 2018).

Da mesma forma, é na indústria que há o maior número de diagnósticos de doenças do sistema osteomuscular e do tecido conjuntivo, ao considerar os auxílios-doença acidentários concedidos pela Previdência Social no Brasil (Carvalho, 2013). Não obstante, os trabalhadores afastados com essas patologias precisam conviver com as limitações para realizar as atividades simples do cotidiano, pois apresentam sintomas dolorosos (Garcia, Vieira \& Garcia, 2013; Alencar \& Nobre, 2017).

Dito isso, nesse estudo verificou-se que as queixas mais frequentes foram: dor (100\%), dormência (65\%), câimbra (38,3\%), choques $(36,6 \%)$, falta de força $(93,3 \%)$, formigamentos $(58,3 \%)$, edema $(45 \%)$, cansaço $(1,7 \%)$, queimação $(1,7 \%)$, deslocamentos $(1,7 \%)$ e latejamento (1,7\%). Do mesmo modo, outros estudos (Guasti et al., 2011; Alencar \& Nobre, 2017; Rosa \& Quirino, 2018; Caieiro et al., 2019) destacam a prevalência elevada de dor osteomuscular nas amostras de trabalhadores estudadas.

Essa sintomatologia de dor pode ser retroalimentada por manifestações de ordem psíquica que acometem os pacientes com DORT, e, com o agravamento do quadro, pode haver a piora dos sintomas psíquicos, levando, assim, a um ciclo vicioso de dor e sofrimento mental em indivíduos que muitas vezes continuam exercendo suas atividades adotando posturas inadequadas (Salermo, Silvestre, \& Sabino, 2011; Fernandes et al., 2016).

Desse modo, tanto as posturas inadequadas, quanto a repetitividade e o alto índice de esforço durante a execução das atividades (Guasti et al., 2011) determinam o alto risco ergonômico que aponta para a necessidade de intervenção nesses fatores ocupacionais (Carvalho, Brasileiro \& Lima, 2019), em busca da promoção e da prevenção da saúde do trabalhador (Freitas et al., 2015) podendo contribuir para o aparecimento da morbidade musculoesquelética em múltiplos locais do corpo (Guasti et al., 2011).

Nesse sentido, esse estudo constatou que as regiões do corpo mais acometidas foram os ombros $(43,8 \%)$ e a coluna lombar (22\%), essa informação pode estar relacionada ao número prevalente de trabalhadores na área industrial, que apresentam funções laborais com maior sobrecarga nos membros superiores e na coluna lombar (Kottke et al., 1986).

Os dados apresentados estão de acordo com os estudos de Duda et al. (2014), no qual as regiões mais acometidas foram ombro, coluna vertebral e punho, e, de Reis et al. (2020), cujas maiores queixas dolorosas e alterações posturais apresentaram-se na coluna vertebral, nos ombros e joelhos. Vale salientar que as articulações do ombro e da coluna lombar, apresentam grandes amplitudes de movimentos, sendo a primeira biomecanicamente instável e a segunda recebe grande sobrecarga de peso (Kottke et al., 1986).

Adicionalmente, estudos mostraram o desenvolvimento de dor lombar em diversas ocupações nas quais os laboriosos permaneceram longos períodos em posturas estáticas (Gallagher \& Callaghan, 2015; Karakolis, Barrett \& Callaghan, 2016) e fazendo o uso excessivo de força física (Graves et al., 2015).

Ainda, no que diz respeito ao surgimento dos sintomas em trabalhadores com DORT, observou-se que 88,3\% vêm sentindo sintomatologia osteomioarticular há menos de cinco anos, esse achado discorda com os de Genovese (1991), que verificou serem esses sintomas e sinais só percebidos quando os indivíduos se encontram em estágio irreversível desses distúrbios.

Bem como, Genovese (1991) explica que isso ocorre porque, geralmente, os sinais e sintomas advindos com os DORT, só se manifestam após longo período de função trabalhista (Salermo, Silvestre \& Sabino, 2011). E, por isso, a avaliação do ambiente laboral deve considerar todo o processo de trabalho e sua relação com os distúrbios, permitindo a formulação de hipóteses de causalidade e de estratégias de prevenção (Caieiro et al., 2019).

Assim, não só as ações de prevenção devem ocorrer, como também, o diagnóstico precoce a partir da realização de exames de imagem. Nesse estudo, destaca-se o fato dos exames serem recentes, visto que $41 \%$ dos entrevistados os fizeram há 
menos de três meses da realização das entrevistas. E, dos 93,3\% indivíduos que levaram os seus exames, 38\% eram ultrassonografia e $36,5 \%$ ressonância magnética.

Isto se deve ao fato do primeiro exame ser solicitado rotineiramente para alterações os ombros, já o segundo para alterações na coluna vertebral, como também, essas regiões foram as que apresentaram maiores incidências das queixas osteomiarticulares por parte dos trabalhadores.

Também, convém dizer, que há coerência na associação realizada entre os exames e as estruturas alteradas, pois o tendão se revelou com maior frequência nas ultrassonografias, e os discos vertebrais nas ressonâncias (Paavola et al., 2002; Barbosa et al., 2002; Amorim et al., 2006).

Além disso, o exame de ultrassonografia mostrou-se bastante sensível, uma vez que a região mais acometida pelas queixas osteomiarticulares foi o ombro e 83,3\% desses exames mostraram alterações nesta região. Em concordância, a pesquisa realizada por Gonzalez (2005), aponta ser a ultrassonografia a melhor escolha para o diagnóstico de patologias nos ombros, porém, para as alterações nos cotovelos, antebraços e punhos ela mostrou baixa sensibilidade.

Mas, vale destacar, que a ultrassonografia é uma importante aliada para a identificação de alterações musculoesqueléticas a partir de algum tempo do início do quadro clínico (Pereira, 2015). Contudo, para o diagnóstico clínico de DORT, a ultrassonografia tem a desvantagem de ser um pouco limitada, já a ressonância magnética, mostra-se $95 \%$ mais sensível e específica no diagnóstico de algumas tendinites (Paavola et al., 2002; Barbosa et al., 2002; Amorim et al., 2006).

Ainda, no estudo de Gonzalez (2005), o serviço de saúde onde foi realizada a pesquisa, teve a necessidade de procurar um exame complementar de baixo custo que suprisse esta dificuldade que havia com as ultrassonografias, na qual as áreas afetadas, por inflamação, teriam uma maior captação de radiofármaco, como na cintilografia óssea, todavia, ela mostrou-se inespecífica para avaliar sintomatologia dolorosa em membros superiores.

No que se refere ao uso da cintilografia óssea trifásica, ela é importante para mostrar anormalidades secundárias aos DORT, que poderiam afetar tecidos próximos e ossos macios, porém não deve ser utilizada para o diagnóstico primário por causa de sua baixa sensibilidade (Amorim et al., 2006). Do mesmo modo, os exames radiográficos apresentou uma baixa sensibilidade e alta especificidade para diagnosticar os DORT, pelo fato da maioria dos resultados serem negativos (Graves $e t$ al., 2015).

Além disso, existem outros métodos eficazes para auxiliar no diagnóstico dos DORT, no estudo de Jesus Filho et al. (2014), a eletroneuromiografia revelou ser um exame complementar valioso para o diagnóstico da síndrome do túnel do carpo, pois mostrou sensibilidade superior à da ultrassonografia e do exame físico. Outro exame que está ganhando cada vez mais espaço no apoio diagnóstico, avaliação e acompanhamento dos quadros sintomáticos dessas patologias, é a termografia, que tem alta sensibilidade, sobretudo, em punho, cotovelo e ombro (Trotta \& Ulbricht, 2015).

Nesse sentido, acerca dos diagnósticos identificados nos exames, foram encontrados os seguintes: tendinopatias subescapular, supra-espinhal, infraescapular, calcária, síndrome do manguito rotador e/ou osteoartrose acromioclavicular $(31,7 \%)$, seguido dos transtornos nos discos vertebrais $(28,1 \%)$.

Dessa forma, as alterações patológicas mais descritas nos tendões, bursas, sinóvias e músculos foram as inflamatórias $(10,3 \%)$ e as calcificações (11,2\%); e, nos discos vertebrais foram as protusões discais (13,1\%) e os osteófitos (10,3\%). E, a estrutura com maior número de alterações descritas foi o tendão $(40 \%)$. Concordando com o parágrafo acima, já que as tendinopatias foram as patologias mais apresentadas pelos entrevistados.

Portanto, as informações apresentadas estão de acordo com os dados Observatório de Saúde e Segurança do Trabalho (2018), pois, considerando os afastamentos do trabalho por doença do tipo acidentário em 2018, ou seja, aqueles concedidos aos indivíduos que sofreram acidente de trabalho ou acometidos de doença profissional, os diagnósticos específicos mais frequentes segundo os códigos da Classificação Internacional de Doenças (CID) e excluindo-se as fraturas, foram as 
dorsalgias, as lesões do ombro, as sinovites e tenossinovites, as mononeuropatias dos membros superiores e os transtornos dos discos vertebrais. Além disso, os achados deste estudo corroboram com os de Duda et al. (2014), que evidenciaram serem as sinovites, tenossinovites e lesões no ombro as afecções relacionadas ao trabalho de maior prevalência.

Por fim, esta pesquisa tem a limitação relacionada ao número de atendimentos realizados no CEREST, que pode não ser condizente com o número de trabalhadores com queixas clínicas de DORT na região, tendo em vista a baixa procura à rede pública de saúde em virtude dos distúrbios, e quando a fazem, nem sempre o profissional de saúde que realiza o primeiro atendimento faz o nexo da sintomatologia com a atividade ocupacional, o que permitiria a realização da notificação do agravo e dos encaminhamentos devidos.

\section{Conclusão}

Diante do exposto, este estudo demonstrou que nos trabalhadores acompanhados pelo Centro de Referência à Saúde do Trabalhador do Cabo de Santo Agostinho, o gênero mais acometido foi o masculino e a idade prevalente mostrou-se entre 31 e 40 anos, com um maior acometimento dos profissionais da indústria, entre eles, os operadores de máquina e ajudantes de produção.

Ressalta-se que os trabalhadores mais acometidos estão numa fase bastante produtiva, podendo influenciar significativamente na sua capacidade para o trabalho, dependendo dos fatores de risco presentes, a doença pode agravar ou o trabalhador apresenta sintomatologia leve, e essa cronicidade da lesão, muitas vezes pode levar a diagnósticos tardios.

Outro achado importante, foi que todos os entrevistados apresentaram sintomatologia dolorosa, como também, não houve diferença significativa entre o gênero e a maioria das queixas relatadas. E, a região mais acometida foi o ombro direito, com manifestações sentidas há pelo menos cinco anos, mostrando a evolução rápida da doença na amostra.

Necessita-se esclarecer a respeito da necessidade dos trabalhadores se alternarem entre as atividades laborais diárias com o fim de evitar o uso contínuo e vicioso de um grupo muscular específico, como também, de se estabelecer pausas periódicas para descanso, pois a maioria desses trabalhadores carece mudar a posição do corpo durante o expediente devido a dores no corpo.

Observou-se também, que a ultrassonografia e a ressonância magnética foram os exames de imagem mais utilizados pelos médicos que acompanharam os entrevistados, sendo assim, importantes aliados no diagnóstico de DORT, principalmente de alterações nos tendões e discos vertebrais, respectivamente.

Desse modo, acredita-se que este estudo avança ao investigar as queixas clínicas e os achados de imagem nas doenças osteomusculares relacionadas ao trabalho, auxiliará os profissionais de saúde a conhecerem melhor os riscos predisponentes às lesõe9s, e, a utilizarem todas as informações clínicas apresentadas com o fim de viabilizar a terapêutica mais adequada para restaurar o desempenho biomecânico e psicossocial dos trabalhadores.

No entanto, tendo em vista a relevância do assunto e a insipiência de artigos publicados sobre os diagnósticos de DORT com a finalidade de analisar os exames de imagem, faz-se necessário a realização de pesquisas futuras acerca da temática considerando também os fatores ocupacionais relacionados ao surgimento dos distúrbios nas diversas profissões.

\section{Referências}

Alencar, M. C. B. \& Nobre, T. L. (2017). Adoecimento e sofrimento de trabalhadores acometidos por LER/DORT. Revista de Psicologia. 8(2):8-18. http://www.periodicos.ufc.br/psicologiaufc/article/view/5861

Amorim, B. J., Etchebehere, E. C. S. C., Torre, G. D., Lima, M. C. L., Santos, A. O., Ramos, C. D., Gonzalez, L. R., Oliveira, J. I., \& Camargo, E. E. (2006). Baixa sensibilidade da cintigrafia óssea trifásica no diagnóstico de lesão por esforço repetitivo. São Paulo Medical Journal. 124(3):145-149. http://dx.doi.org/10.1590/S1516-31802006000300007 
Barbosa, M. H. N., Volpon, J. B., Elias, J. R. J., \& Muccillo, G. (2002). Diagnóstico por imagem nas rupturas do manguito rotador. Acta Ortopédica Brasileira. 10(4). https://www.scielo.br/pdf/aob/v10n4/14326.pdf

Besharati, A., Daneshmandi, H., Zareh, K., Fakherpour, A., \& Zoaktafi, M. (2020). Work-related musculoskeletal problems and associated factors among office workers. International Journal of Occupational Safety and Ergonomics. 26(3): 632-638. 10.1080 / 10803548.2018 .1501238

Caieiro, T. T. M., Assis, D. B., Mininel, V. A., Rocha, F. L. R., \& Hortense, P. (2019). Dor osteomuscular: uma comparação entre trabalhadores administrativos e de produção de uma indústria agroavícola. Revista Brasileira de Medicina do Trabalho. 17(1):30-38. 10.5327/Z1679443520190277.

Carvalho, R. (2013). Distúrbios Musculoesqueléticos em Membros Superiores e Pescoço em Trabalhadores da Industria de Calçados [tese]. Salvador: Faculdade de Medicina da Universidade Federal da Bahia.

Carvalho, R. L. R., Brasileiro, F. R. C. P., \& Lima, V. M. C. (2019). Demandas psicológicas, baixo apoio social e repetitividade: fatores ocupacionais associados à dor musculoesquelética de trabalhadores da indústria de calçados. Revista Brasileira de Saúde Ocupacional. [Internet]. 44: e6. http://www.scielo.br/scielo.php?script=sci_arttext\&pid=S030376572019000101304\&lng=en. http://dx.doi.org/10.1590/2317-6369000001517.

Duda, T. M. R., Delbim, L. R., Hunger, M. S., \& Martelli, A. (2014). Doenças osteomusculares relacionadas ao trabalho e à ginástica laboral como estratégia de enfrentamento. Archives of Health Investigation. 3(5):57-65. https://www.archhealthinvestigation.com.br/ArcHI/article/view/796

Fernandes, R. C. P., Pataro, S. M. S., Carvalho, R. B., \& Burdoff, A. (2016). The concurrence of musculoskeletal pain and associated work-related factors: a cross sectional study. BMC Public Health. 16(1):628. 10.1186/s12889-016-3306-4

Freitas, C., Alencar, M. C. B., Lopes, L. V., \& Souza, F. G. (2015). Perfil de sujeitos com transtornos dos tecidos moles atendidos em um serviço de saúde do trabalhador e as LER/DORT. Cadernos de Terapia Ocupacional UFSCar, São Carlos, 23(2):305-312. http://dx.doi.org/10.4322/0104-4931.ctoAO0453

Gallagher, K. M. \& Callaghan, J. P. (2015). Early Static Standing is Associated with Prolonged Standing Induced Low Back Pain. Human Movement Science. 44: 111-121. 10.1016/j.humov.2015.08.019

Garcia, B. T., Vieira, E. B., \& Garcia, J. B. (2013). Relação entre dor crônica e atividade laboral em pacientes portadores de síndromes dolorosas. Revista Dor. 14(3):204-9. http://dx.doi.org/10.1590/S1806-00132013000300011.

Genovese, W. \& Lopes, A. (1991). Doenças profissionais do cirurgião dentista. Pancast.

Graves, L., Murphy, R., Shepherd, S. O., Cabot, J., \& Hopkins, N. D. (2015). Evaluation of sit-stand workstations in an office setting: a randomised controlled trial. BMC Public Health. 15:1145. https://doi.org/10.1186/s12889-015-2469-8

Guasti, L. M., Rocha, N. G., Brentini, A. L., Ramos, V. E., \& Veiga, Q. P. R. (2011). Riscos de lesão musculoesquelética em diferentes setores de uma empresa calçadista. Fisioterapia e Pesquisa. 18(3):252-257. https://doi.org/10.1590/S1809-29502011000300009.

Jesus Filho A. G., Nascimento, B. F., Amorim, M. C., Naus, R. A. S., Loures, E. A., \& Moratelli, L. (2014). estudo comparativo entre o exame físico a eletroneuromiografia e a ultrassonografia no diagnóstico da síndrome do túnel do carpo. Revista Brasileira de Ortopedia, 49(5):446-51.

Karakolis, T., Barrett, J., \& Callaghan, J. P. (2016). A Comparison of Trunk Biomechanics, Musculoskeletal Discomfort and Productivity during Simulated Sit-stand Office Work. Ergonomics. 59(10): 1275-1287. 10.1080/00140139.2016.1146343

Kottke, F. J. et al. (1986). Tratado de medicina física e reabilitação. Manole.

Lida, I., \& Guimarães, L. B. M. (2016). Ergonomia: projeto e execução. (3a ed.), Edgard Blücher.

Lopes, S. A. P., Pelai, E. B., Foltran, F. A., Bigaton, D. R., \& Teodori, R. M. (2017). Risco ergonômico e distúrbio osteomuscular relacionado ao trabalho em trabalhadores de fabricação de máquinas e equipamentos. Cadernos Brasileiros de Terapia Ocupacional, São Carlos, 25(4): 743-750. http://dx.doi.org/10.4322/2526-8910.ctoAO0961

Ministério da Saúde. (2019). Saúde Brasil 2018 uma análise da situação de saúde e das doenças e agravos crônicos: desafios e perspectivas. Ministério da Saúde, Secretaria de Vigilância em Saúde, Departamento de Vigilância de Doenças e Agravos Não Transmissíveis e Promoção da Saúde - Brasília: Ministério da Saúde, 424.http://bvsms.saude.gov.br/bvs/publicacoes/saude_brasil_2018_analise_situacao_saude_doencas_agravos_cronicos_desafios_perspectivas.pdf

Ministério da Saúde. (2012). Dor relacionada ao trabalho: lesões por esforços repetitivos (LER): distúrbios osteomusculares relacionados ao trabalho (Dort). Secretaria de Vigilância em Saúde Departamento de Vigilância em Saúde Ambiental e Saúde do Trabalhador https://bvsms.saude.gov.br/bvs/publicacoes/dor_relacionada_trabalho_ler_dort.pdf

Ministério da saúde. (2001). Doenças relacionadas ao trabalho: Manual de procedimentos para os serviços de saúde. Brasília: Ministério da Saúde. https://www.paho.org/bra/index.php?option=com_docman\&view=document\&layout=default\&alias=207-doencas-relacionadas-ao-trabalho-manualprocedimentos-para-os-servicos-saude-7\&category_slug=saude-e-ambiente-707\&Itemid=965

Observatório de Saúde e Segurança do Trabalho. (2018). Perfil dos Afastamentos por Agravo de 2012 à 2018 - Instituto Nacional do Seguro Social (INSS). SmartLab. Observatório de Segurança e Saúde no Trabalho. https://smartlabbr.org/sst/localidade/26?dimensao=frequenciaSinan

Orange, N. O., Wallis, B., Roer, K., Archer-Heese, G., \& Aguilar, Z. (2016). Do personal factors or types of physical tasks predict workplace injury? Work Heal Saf. 64(4):141-51. https://pubmed.ncbi.nlm.nih.gov/27056936/ Doi:10.1177/2165079916630552.

Organização Internacional do Trabalho. (2018). Estatística mundial: o enorme fardo de más condições de trabalho: OIT. http:/www.ilo.org/moscou/áreasdetrabalho/segurançaesaúdeocupacional/WCMS_249278/idioma/indice.htm

Paavola, M., Kannus, P., Jarvinen, T. A. H., Khan, K., Józsa, L., \& Jarvinen, M. (2002). Current concepts review: achiles tendinopathy. The Journal of Bone \& Joint Surgery. 84-A (11): 2062-2076. https://portalbiocursos.com.br/ohs/data/docs/56/22-_Achilles_Tendinopathy.pdf 
Research, Society and Development, v. 10, n. 1, e36710111865, 2021

(CC BY 4.0) | ISSN 2525-3409 | DOI: http://dx.doi.org/10.33448/rsd-v10i1.11865

Pereira, L. F. (2015). Ultrassonografia na Investigação das Lesões Musculoesqueléticas da LER/DORT. Revinter.

Pereira, A. S. et al. (2018). Metodologia da pesquisa científica. [e-book]. Santa Maria. Ed. UAB/NTE/UFSM. https://repositorio.ufsm.br/bitstream/handle/1/15824/Lic_Computacao_Metodologia-Pesquisa-Cientifica.pdf?sequence=1

Reis, V. F., Alves, F. R. F., Moraes, M. M., Paiva, T. G. A., Guimarães, A. C., Chiavegato Filho, L. G., \& Damázio, L. C. M. (2020). Incidência de lesões musculoesqueléticas entre os trabalhadores dos serviços gerais do campus Dom Bosco da Universidade Federal de São João Del-Rei. Biológicas \& Saúde. 10(34):33-6. https://doi.org/10.25242/8868103420202030

Rosa, M. A. G., \& Quirino, R. (2018). Ergonomia, saúde e segurança no trabalho: interseccionalidade com as relações de gênero. CIENTEC- Revista de Ciência, Tecnologia e Humanidades do IFPE. 9(3). http://revistas.ifpe.edu.br/index.php/cientec/article/view/156

Salerno, V. L., Silvestre, M. P., \& Sabino, M. O. (2011). Interfaces LER/Saúde Mental: a experiência de um Centro de Referência em Saúde do Trabalhador do Estado de São Paulo. Revista Brasileira de Saúde Ocupacional. 36(123):128-38. http://dx.doi.org/10.1590/S0303-76572011000100012.

Silva Júnior, J. S., Buzzoni, G. P., \& Morrone, L. C. (2016). Queixas osteomusculares dos trabalhadores e condições biomecânicas no trabalho em metalúrgica de alumínio. Revista Brasileira de Medicina do Trabalho. 14(2):115-9. http://www.rbmt.org.br/details/39/pt-BR/queixas-osteomusculares-dos-trabalhadorese-condicoes-biomecanicas-no-trabalho-em-metalurgica-de-aluminio

Silva, P. L. N., Monção, M. J. D., Oliveira, B. L. S., Cardoso, T. V., Soares, L. M., \& Costa, A. A. (2019). Distúrbio osteomuscular relacionado ao trabalho: identificação dos fatores socioeconômicos e clínicos autorreferidos por trabalhadores de saúde de uma instituição hospitalar do município de Espinosa, Minas Gerais, Brasil. Revista Rede de Cuidados de Saúde. 13(1), 9-20. http://fi-admin.bvsalud.org/document/view/m52vs

Skovlund, S. V., Bláfoss, R., Sundstrup, E., \& Andersen, L. L. (2020). Association between physical work demands and work ability in workers with musculoskeletal pain: cross-sectional study. BMC Musculoskelet Disord. 21, 166. https://doi.org/10.1186/s12891-020-03191-8

Sousa, F. C. A., Miranda Júnior, R. N.C., Siqueira, H. D. S., Silva, W.C., Sousa, J. P. S., \& Pinho, R. S. (2020). Comportamento de biomarcadores em professores de ensino médio público. Research, Society and Development, 9(1), e64911616. https://doi.org/10.33448/rsd-v9i1.1616

Trindade, L. L., Araldi, A., Ferraz, L., Amestoy, S. C., Vendruscolo, C., Bordignon, M. (2014). Perfil dos segurados em benefício do auxílio-doença acidentário na agência da previdência social de Xanxerê/SC. Revista de Terapia Ocupacional da Universidade de São Paulo. 25(3), 272-278. http://dx.doi.org/10.11606/issn.2238-6149.v25i3p272-278

Trotta, J., \& Ulbricht L. (2015). Thermography in Complementary Diagnostic of Musculoskeletal Diseases. Pan American Journal of Medical Thermology. 2(1), 7-13. http://dx.doi.org/10.18073/2358-4696/pajmt.v2n1p7-13

Zangirolami-Raimundo, J., Echeimberg, J. O., \& Leone, C. (2018). Research methodology topics: Cross-sectional studies. Journal of Human Growth and Development, 28(3):356-360. http://dx.doi.org/10.7322/jhgd.152198 